\title{
Confucianism and the East Asian Miracle
}

\author{
By Ming-Yih Liang
}

\section{Appendix}

This appendix contains proofs and derivations of Propositions 1-3 in the main text.

The aggregate dynamics of the economy can be conveniently described by a system of deterministic differential equations involving the variables $C_{1}, C_{2}, N_{11}$ and $N_{12}$. Maximization of utility by the two representative households results in the familiar Euler equations (11) and (21). As in the text, we denote the two constant growth rates of $C_{1}$ and $C_{2}$ as $\gamma_{C 1}$ and $\gamma_{C 2}$ where $\gamma_{C 2}>\gamma_{C 1}$ because $\rho_{2}<\rho_{1}$. The striking aspect of equations (11) and (21) is that consumption growths do not depend on the number of intermediates, $N s$.

To study the dynamic behavior of $N_{11}$ and $N_{12}$, we must solve the system of simultaneous differential equations (14) and (20). The equality between $w_{1}$ and the marginal product of labor implies

$$
w_{1}=(1-\alpha) \cdot\left(Y_{1} / L_{1}\right) \text {. }
$$

After some manipulation, the interest rate, given by equations (7) and (8) can be written as

$$
r_{1}=(1 / \eta) \cdot(1-\alpha) \cdot \alpha \cdot\left(Y_{1} / N_{1}\right)
$$

Hence, aggregate income, $w_{1} L_{1}+r_{1} \eta_{1} N_{1}$, equals $Y_{1}-\alpha^{2} Y_{1}$. It follows that the Western household's budget constraint in equation (14) becomes

$$
\begin{aligned}
\eta \dot{N}_{11} & =Y_{1}-\alpha^{2} Y_{1}-C_{1}-r_{1} \eta N_{12} \\
& =\left(1-\alpha^{2}\right) Y_{1}-C_{1}-r_{1} \eta N_{12} \\
& =(1+\alpha)(1-\alpha) Y_{1}-C_{1}-r_{1} \eta N_{12} .
\end{aligned}
$$

If we substitute for $Y_{1}$ and $r_{1}$ from equations (5) and (8) into (A3) and also use equation (7), we get a formula for the Western household's budget constraint 


$$
\eta \dot{N}_{11}=\left[\pi_{1} \cdot(1+\alpha) / \alpha\right] \cdot N_{11}+\left(\pi_{1} / \alpha\right) \cdot N_{12}-C_{1} .
$$

By a similar process of substitution into equation (20), using equations (5), (6), (7), (8) and (18), we can also get a formula for the Eastern household's budget constraint

$$
\begin{aligned}
(v \lambda+\eta) \dot{N}_{12}= & {\left[\lambda \pi_{2} \cdot(1+\alpha) / \alpha+\pi_{1}\right] \cdot N_{12} } \\
& +\left[\lambda \pi_{2} \cdot(1+\alpha) / \alpha\right] \cdot N_{11}-v \lambda \dot{N}_{11}-C_{2} .
\end{aligned}
$$

We now must solve the system of simultaneous differential equations (A4) and (A5). We can substitute $\left(\lambda \pi_{2} / \pi_{1}\right) \cdot(\mathrm{A} 4)$ into equation (A5) to get

$$
\dot{N}_{12}=r_{1} N_{12}+a \cdot C_{1}-b \cdot C_{2},
$$

where $a$ and $b$ are defined as

$$
\begin{aligned}
& a=v \lambda /[(v \lambda+\eta) \cdot \eta], \\
& b=1 /(v \lambda+\eta) .
\end{aligned}
$$

(A5) is a first-order, linear differential equation in $N_{12}$. The general solution of this equation is

$$
N_{12}(t)=(\text { constant }) \cdot e^{r_{1} t}-\left[a /\left(r_{1}-\gamma_{C 1}\right)\right] \cdot C_{1}(t)+\left[b /\left(r_{1}-\gamma_{C 2}\right)\right] \cdot C_{2}(t) .
$$

We assume that the production function is sufficiently productive to ensure growth in consumption, but not so productive as to yield unbounded utility

$$
r_{1}>\rho_{2}>r_{1} \cdot(1-\theta) \text {. }
$$

The first part of this condition guarantees that $\gamma_{C_{2}}>0$. The second part ensures that the attainable utility is bounded and implies that $r_{1}-\gamma_{C_{2}}>0$. The transversality condition for the dynamic optimization by the Eastern household implies

$$
\lim _{t \rightarrow \infty}\left\{N_{12}(t) \cdot e^{-r_{1} t}\right\}=0 \text {. }
$$

If we substitute for $N_{12}(t)$ from equation (A7) into the transversality condition in equation (A9), we get 
(A10)

$$
\begin{array}{r}
\lim _{t \rightarrow \infty}\left\{\text { constant }-\left[a /\left(r_{1}-\gamma_{C 1}\right)\right] \cdot C_{1}\left(T_{0}\right) \cdot e^{-\left(r_{1}-\gamma_{C 1}\right) t}\right. \\
\left.+\left[b /\left(r_{1}-\gamma_{C 2}\right)\right] \cdot C_{2}\left(T_{0}\right) \cdot e^{-\left(r_{1}-\gamma_{C 2}\right) t}\right\}=0 .
\end{array}
$$

Since $C_{1}\left(T_{0}\right)$ and $C_{2}\left(T_{0}\right)$ are finite and $r_{1}-\gamma_{\mathrm{C} 2}>0, r_{1}-\gamma_{\mathrm{C} 1}>0$, the second and the third terms in the braces converge toward zero. Hence, the transversality condition requires the constant to be zero. The solution of $N_{12}$ becomes

$$
N_{12}(t)=-\left[a /\left(r_{1}-\gamma_{C 1}\right)\right] \cdot C_{1}(t)+\left[b /\left(r_{1}-\gamma_{C_{2}}\right)\right] \cdot C_{2}(t) .
$$

If we substitute $N_{12}(t)$ from equation (A11) into equation (A4), we get

$$
\dot{N}_{11}=\left[r_{1} \cdot(1+\alpha) / \alpha\right] \cdot N_{11}-f \cdot C_{1}+g \cdot C_{2},
$$

where $f$ and $g$ are defined as

$$
\begin{aligned}
& f=a \cdot r_{1} /\left[\alpha \cdot\left(r_{1}-\gamma_{c_{1}}\right)\right]+1 / \eta, \\
& g=b \cdot r_{1} /\left[\alpha \cdot\left(r_{1}-\gamma_{c_{2}}\right)\right] .
\end{aligned}
$$

(A12) is a first-order, linear differential equation in $N_{11}$. By repeating the same procedure as above, we can solve for $N_{11}$ as

$$
\begin{aligned}
N_{11}(t)= & \left.\left\{f /\left[r_{1} \cdot(1+\alpha) / \alpha-\gamma_{C 1}\right)\right]\right\} \cdot C_{1}(t) \\
& \left.-\left\{g /\left[r_{1}(1+\alpha) / \alpha-\gamma_{C 2}\right)\right]\right\} \cdot C_{2}(t)
\end{aligned}
$$

Finally, if we add (A11) and (A13) together, we get

$$
\begin{aligned}
N_{1}(t) & =N_{11}(t)+N_{12}(t) \\
& =m \cdot C_{1}(t)+n \cdot C_{2}(t)
\end{aligned}
$$

where $m$ and $n$ are defined as

$$
\begin{aligned}
& m=b /\left[r_{1} \cdot(1+\alpha) / \alpha-\gamma_{c_{1}}\right], \\
& n=b /\left[r_{1} \cdot(1+\alpha) / \alpha-\gamma_{c_{2}}\right] .
\end{aligned}
$$

The transversality conditions imply that $r_{1}-\gamma_{C_{1}}>0$ and $r_{1}-\gamma_{C_{2}}>0$, and hence $\left[r_{1} \cdot(1+\alpha) / \alpha\right]-\gamma_{c 1}>0,\left[r_{1} \cdot(1+\alpha) / \alpha\right]-\gamma_{c_{2}}>0$. Since $a, b, f, g, m, n>$ 0 , the dynamic analysis of $N_{12}, N_{11}$ and $N_{1}$ as described in the text can be derived from the 
following three theorems of real numbers:

Let $A, B, C$ be real numbers and functions of time, $t . A\left(T_{0}\right), B\left(T_{0}\right), C\left(T_{0}\right)>0$ at $t=T_{0}$.

Theorem 1: If $A=B-C$, and both $B$ and $C$ grow at positive constant rates with $\dot{B} / B>\dot{C} / C$, then $\dot{A} / A>\dot{B} / B$ initially and $\dot{A} / A$ declines monotonically toward $\dot{B} / B$ as $t \rightarrow \infty$.

Theorem 2: If $A=B-C$, and both $B$ and $C$ grow at positive constant rates with $\dot{B} / B<\dot{C} / C$, then $\dot{A} / A<\dot{B} / B$ initially and $\dot{A} / A$ declines monotonically. Let $\dot{A} / A>0$ initially, then $\dot{A} / A$ declines until at the some point, $t=T_{1}>T_{0}, \dot{A} / A=0$ and $A$ begins to decline from then on for $t>T_{1} ; A$ will eventually decline until at $t=T_{2}>T_{1}$, when $B=C, A=0$. Immediately after $t>T_{2}, \dot{A} / A>\dot{C} / C$ and then declines monotonically toward $\dot{C} / C$ as $t \rightarrow \infty$.

Theorem 3: If $A=B+C$, and both $B$ and $C$ grow at positive constant rates with $\dot{B} / B>\dot{C} / C$, then $\dot{B} / B>\dot{A} / A>\dot{C} / C$ initially and $\dot{A} / A$ rise monotonically toward $\dot{B} / B$ as $t \rightarrow \infty$. 